\title{
Dysphagia Of Rare Etiology
}

Tridip Kumar Sengupta ${ }^{*}$, Soumya Mishra ${ }^{1}$, Magna Manjareeka ${ }^{1}$

'Department of Physiology, Kalinga Institute of Medical Sciences, KIIT University, Bhubaneswar, Odisha, India. Abstract

Dysphagia is one of the most common symptoms among the gastrointestinal disorders. Along with this, the mass effect during deglutition in old age is suggestive of malignancy in the oesophagus, mostly in developing country like India. But dysphagia due to left atrial enlargement is extremely uncommon. We present a case of cardiac dysphagia,where dysphagia is due to left atrial enlargement compressing the oesophagus from outside (mechanical obstruction). The patient got relieved after administration of diuretics, which decreased the left atrial pressure and thereby the mechanical compression over the oesophagus was released.

Key Words:Carcinoma Oesophagus, Left atrial enlargement, Cardiac Dysphagia

INTRODUCTION

$\mathrm{C}$ ases with dysphagia, not associated with gastrointestinal disorder leads to diagnosticand treatment dilemmas. The disease in the octogenarian age group leads to the diagnosis of malignancy of oesophagus. But we presenting a case of cardiac dysphagia which was initially confused with gastrointestinal disturbances. We reviewed the literature, and found a very rare similarity with the case we studied in depth.

\section{CASE PRESENTATION}

An 86 years old, lean and thin woman presented with a few months history of feeling of something obstructing inside the throat during deglutition, mainly for solids (mass effect), with difficulty in swallowing (dysphagia). She stated that minimal weight loss was observed within last few months. The patient was on antihypertensive medications and Proton Pump Inhibitors (PPI) for few months to year, prescribed elsewhere.

\section{Examination}

On general clinical examination it was found that her temperature was normal. Pulse was within normal limits. She was on antihypertensive and her blood pressure was normal. She was not significantly anaemic. No palpable lymph gland was detected. On systemic examination of gastrointestinal system it was found that there was mild epigastric tenderness. Apart from a systolic murmur of grade $3 / 6$, heard over the cardiac apical region, there was no apparent abnormality detected on cardiovascular examination. Central nervous system and respiratory system examination revealed no abnormal findings.

\section{Provisional diagnosis: (?) Carcinoma Oesophagus.}

\section{Investigations done}

From the detailed history and clinical examination of the women, considering her old age, carcinoma of the esophagus was suspected and she was advised for examination of total haemoglobin concentration, postero-anterior (PA) view of chest $\mathrm{X}$-ray and Barium swallow oesophagus. The investigation reports showed haemoglobin concentration to be $10.3 \mathrm{gm} \%$. There was enlarged cardiac shadow in her PA view of chest X-ray

\section{Address for correspondence* \\ Dr. Tridip Kumar Sengupta}

Department of Physiology,Kalinga Institute of Medical Sciences, KIIT University, Bhubaneswar - 751024 Odisha, India.E mail id - tridipcardio@gmail.com

Mobile No. - +918763236390 / +919434119791 shown in Figure -1.Figure - 2 shows Barium swallow study of the oesophagus revealing enlargement of left atrium which has displaced the oesophagus posteriorly. Thereafter, a Transthoracic echocardiography was advised to confirm the result of the barium swallow study. The trans-thoracic echocardiography showed a good Left Ventricular Systolic Function with Ejection Fraction (EF) 61\%, Grade II Mitral regurgitation (MR), left atrial enlargement. There was no pulmonary arterial hypertension.

\section{Treatment}

She was prescribed Hydrochlorothiazide, $12.5 \mathrm{mg}$ daily per oral (diuretics) along with her previous medicines i.e. Pantoprazole \& antihypertensive - Ramipril. She improved dramatically within one week. Her complaints of dysphagia and "feeling of something obstructing inside throat" subsided within few days; and gradually her appetite returned to nearly normal.

\section{Follow-up}

Repeat Barium-meal swallow oesophagus examination was done which showed normal contour and normal position of the oesophagus. No mechanical compression over the oesophagus was found shown in Figure - 3. But repeat Transthoracic echocardiographic examination was not done due the financial constraint of the patient.

\section{Final diagnosis: Cardiovascular Dysphagia. \\ DISCUSSION}

Dysphagia due to left atrial enlargement impinging on the esophagus is a rare presentation. This is cardiovascular dysphagia, which is very uncommon clinical presentation. As the left atrium is the midline posterior chamber of the heart, lying just infront of the oesophagus, so, enlargement of former may cause mechanical compression over the oesophagus. Again, posterior displacement of left atrium may cause difficulty in deglutition and mass effect on the oesophagus. The probable cause of the enlargement of left atrium in this case may be due to Mitral Regurgitation (MR). The reduction of MR was achieved by use of diuretics which ultimately ameliorated the dysphagia. For proper evaluation of mitral regurgitation and obviously for treatment for that, the patient was advised to consult with a cardiologist.

A similar type of case was seen who had dysphagia for solid foods for more than one year with weight loss in spite of good appetite. She had a history of myocardial infarction 4 years earlier, complicated with acute mitral regurgitation due to papillary muscle rupture [1].In 1997, an 84 years old woman presented with dysphagia having LA dilation withglobal heart 
Figure - 1. Enlarged cardiac shadow

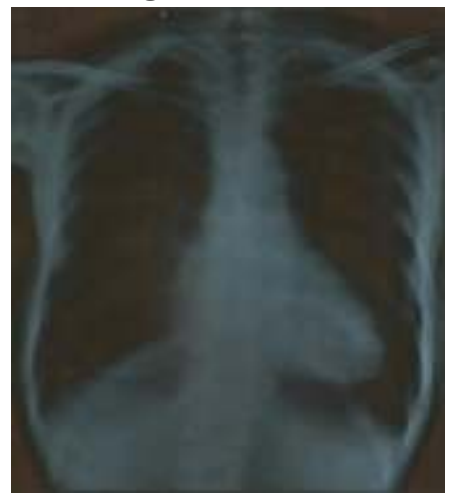

Figure - 2. Posterior

displacement of oesophagus

due to compression by

enlarged left

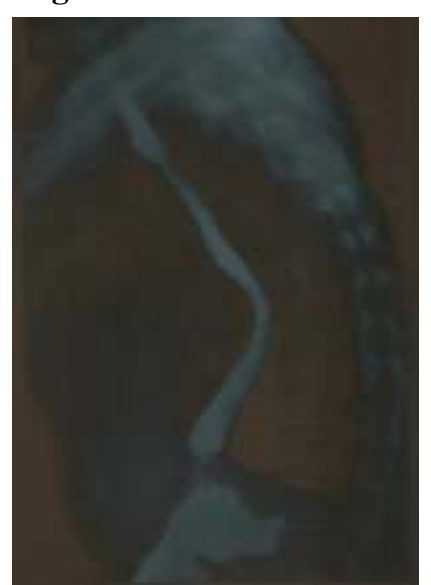

Figure - 3. Normal contour and position of the oesophagus. atrium.

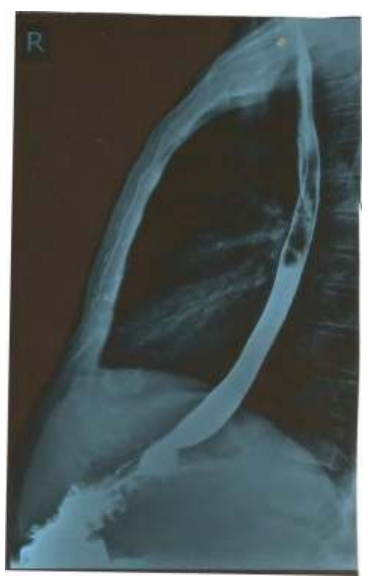

failure [2].

An 82 yrs old woman with a two years history of atrial fibrillation, non-ischemiccardiomyopathy, severe mitral regurgitation, Chronic Obstructive Pulmonary Disease(COPD) and hypertension presented with chest pain showed narrowing of oesophagus from an extrinsic structure on esophagogram. CT scan of chest confirmed the diagnosis of oesophagus compression due to enlarged LA and pulmonary hypertension [3].

In this case the age of the patient is 86 yrs with complaint of dysphagia for few months only. She was hypertensive and was on antihypertensive and PPI for acid-peptic disorder for few months to years. The patient was referred to a Cardiologist for further evaluation of MR. Though uncommon, but the take home message is "suspicion should be present in mind".

\section{REFERENCES}

1. Gotsman I,Mogle P, Shapira MY. "An Unusual Cause of Dysphagia". POST GRADUATE MEDICAL JOURNAL.1995; 75(888): 629-631.

2. Kress S, Martin WR, Berz C, Reimann JE. " Dysphagia Secondary to left atrial dilatation". Z Gastroenterol. 1997; 35(11):1007-11.

3. Khan FA, Rasid Y, Khosrani F, Atallah C, Efrusy M, "Dysphagia Megalatriensis Caused by Enlarged Left Atrium and Pulmonary Vein", Program No.P29, ACG 2012,October 21, Annual Scientific Meeting Abstract, Las Vegas,NV; American College of Gastroenterology. 